\title{
PALOMA-3 clinical trial: is there a significant benefit in overall survival for breast cancer? Is it worth it?
}

\author{
Jacob J Adashek ${ }^{1}$, Pedro N Aguiar Jr²,3, Pedro Castelo-Branco ${ }^{4,5}$, Gilberto L Lopes Jr ${ }^{6}$ \& \\ Ramon Andrade de Mello*,7,8,9 \\ ${ }^{1}$ Western University of Health Sciences, College of Osteopathic Medicine of the Pacific, Pomona, CA, USA \\ ${ }^{2}$ Faculdade de Medicina do ABC, Santo André, Brazil \\ ${ }^{3}$ Américas Centro de Oncologia Integrado, São Paulo, Brazil \\ ${ }^{4}$ Department of Biomedical Sciences \& Medicine, Division of Oncology, University of Algarve, 8005-139, Faro, Portugal \\ ${ }^{5}$ Algarve Biomedical Center, Campus Gambelas, Edificio 2, 8005-139, Faro, Portugal \\ ${ }^{6}$ Sylvester Comprehensive Cancer Center at the University of Miami, Miami, FL, USA \\ ${ }^{7}$ Department of Medical Oncology, Federal University of São Paulo, São Paulo, Brazil \\ ${ }^{8}$ Division of Medical Oncology, Hospital 9 de Julho, São Paulo, Brazil \\ ${ }^{9}$ Departamento de Ciências Biomédicas e Medicina, Universidade do Algarve, Faro, Portugal \\ *Author for correspondence: Tel.: +5511 950835 698; ramondemello@gmail.com
}

"Breast cancer is a very important disease in terms of public health worldwide. Several patients are diagnosed and follow to a progressive disease every year. CDK4/6 inhibitors (CDKi) were a paradigm shift in the treatment of hormone receptor-positive $(\mathrm{HR}+)$, HER2-negative advanced breast cancer $(\mathrm{aBC})$." $^{\prime}$

First draft submitted: 25 January 2019; Accepted for publication: 15 March 2019; Published online: 8 May 2019

Keywords: CDK inhibitors $\bullet$ hormone-positive HER-2-negative advanced breast cancer $\bullet$ overall survival • Phase III

Breast cancer is a very important disease in terms of public health worldwide. Several patients are diagnosed and follow to a progressive disease every year. CDK4/6 inhibitors (CDKi) were a paradigm shift in the treatment of hormone receptor-positive (HR+), HER2-negative advanced breast cancer $(\mathrm{aBC})$ [1].

Historically, the treatment of HR+/HER2- advanced breast cancer was solely the blockade of estrogen receptor signaling, also called endocrine therapy (ET) [2]. Unfortunately, almost all tumors developed resistance to ET [3]. The recognition that many cases of HR+/HER2- breast tumors have a cyclin D1 and a CDK4 amplification led to the development of $\mathrm{CDKi}$ as a potential mechanism to overcome resistance to ET [4]. These innovative approaches faced a new era in terms of aBC outcomes.

Palbociclib, ribociclib and abemaciclib are three CDKi approved by the US FDA for the treatment of aBC. When combined with ET all of these agents have shown a statistically significant benefit in terms of progressionfree survival (PFS) compared with ET alone, not only for patients previously treated with anti-estrogen therapies, but also in treatment-naive patients [5-12]. However, it is not completely known if the addition of CDKi to ET can also improve overall survival (OS) compared with ET alone.

Recently, the OS results from the PALOMA-3 trial were published. In this trial, patients who had progressed or relapsed on ET alone were randomized to second-line fulvestrant or fulvestrant + palbociclib. The combination improved the median OS by 6.9 months (from 28.0 months with fulvestrant vs 34.9 months with fulvestrant + palbociclib), although it did not reach statistical significance (HR: 0.81;95\% CI: 0.64-1.03; p = 0.09) [13]. This treatment combination was well tolerated and clinically meaningful for experienced oncologists.

The Phase II PALOMA-1 trial compared palbociclib with letrozole to letrozole alone for patients with previously untreated HR+/HER2- advanced breast cancer. In this trial, the median PFS was 20.2 months (95\% CI: $13.8-$ 27.5) for the palbociclib with letrozole and 10.2 months (5.7-12.6) for the letrozole alone group (HR: 0.488; $95 \%$ CI: $0.319-0.748$; one-sided $\mathrm{p}=0.0004)$ [5]. This landmark study led to the accelerated approval of palbociclib 
with letrozole in advanced HR+/HER2- breast cancer by the FDA. After a median follow-up of 64.7 months, the median OS was 37.5 months (95\% CI: 31.4-47.8) for the palbociclib with letrozole and 34.5 months (95\% CI: 27.4-42.6) for the letrozole alone group (HR: 0.897; 95\% CI: 0.623-1.294; one-sided p = 0.281) [14]. Palbociclib + letrozole demonstrated to be well tolerated to patients and also easy to use in the clinical practice since those drugs are both per os and are clinically meaningful. However, QTc interval was an issue to be considered on this combination.

After PALOMA-1, a Phase III trial (PALOMA-2) was developed in order to confirm the benefit of palbociclib + letrozole over letrozole alone for patients with previously untreated HR+/HER2- advanced breast cancer. In the PALOMA-2 trial, palbociclib + letrozole improved PFS by $35 \%$ compared with letrozole + placebo (median PFS by central review 30.5 vs 19.3 months, respectively) [6]. Unfortunately, the PALOMA-2 OS data were still immature when the study was published.

The Phase III MONALEESA-2 trial recently reported updated results including OS data. In the MONALEESA2 trial, patients with previously untreated HR+/HER2- advanced breast cancer were randomized to receive either ribociclib + letrozole versus placebo + letrozole. The median PFS was updated and showed an increase from 9.3 to 16.0 months (95\% CI: $13.4-18.2)$ for the letrozole alone group and to 25.3 months (95\% CI: $23.0-30.3$ ) in the ribociclib and letrozole cohort [15]. After 26.4 months of follow-up, MONALEESA-2 reported that the median OS was not reached (95\% CI not reached) for the ribociclib + letrozole and 33.0 months (95\% CI: 33.0-not reached) for the placebo + letrozole group (HR: 0.746; 95\% CI: 0.517-1.078). Even though the OS was still immature as there were few OS events among both arms (50 events among 334 patients in the ribociclib + letrozole arm and 66 events among 334 patients in the placebo + letrozole arm) [15]. This treatment combination was also well tolerated by the patients and was clinically meaningful. Also, the per os presentation allows to adjust treatment dose while considerable adverse event leading to more patient treatment adhesion.

The updated OS data proposes several questions that the authors here hope to answer: combining all available data regarding $\mathrm{CDKi}+\mathrm{ET}$ is there a statistical significant benefit compared with ET alone? Is CDKi $+\mathrm{ET}$ a cost-effective treatment? Is there a particular subgroup of patients who will benefit most for whom CDKi + ET might be cost effective?

We developed a pooled meta-estimate to assess the OS results from MONALEESA-2, PALOMA-1 and PALOMA-3 [13-15]. Combining the results for first- and second-line, CDKi + ET improved OS by 19\% (HR: $0.81 ; 95 \%$ CI: $0.68-0.97 ; \mathrm{p}=0.02)$. Even though the OS gain is not statistically significant when considering first-line (HR: 0.82; 95\% CI: 0.63-1.06; p = 0.13) and second-line (HR: 0.81; 95\% CI: 0.64-1.03; p = 0.08), separately. Supplementary Figure 1 summarizes the pooled OS meta-estimate.

Another important aspect is whether the utilization of CDKi + ET is cost effective in the treatment of $\mathrm{HR}+/ \mathrm{HER} 2-\mathrm{aBC}$. The average retail cost of palbociclib in USA is US $\$ 12,863.79$, which means that 12 cycles, as the median number of cycles in the PALOMA-3 trial, would equate to US $\$ 154,365.48$ per patient [16]. To be considered cost effective with a willingness to pay of US $\$ 100,000$ per quality-adjusted life year (QALY), CDKi + ET would need to provide at least 1.54 more years of life (18.48 months) compared with ET alone [17]. However, in the PALOMA-3 trial, the median improvement in OS was 6.9 months, meaning that the use of palbociclib in the second-line setting would not be cost effective at the current price.

In a subgroup analysis of the PALOMA-3 trial, three specific groups derived the greatest benefit in OS: patients with previous sensitivity to ET (10 months), patients without visceral metastasis (11.5 months), patients free of disease after adjuvant treatment $>24$ months ( 9.8 months) and patients with ESR1 mutations (11 months) [13]. The hazard ratios for death for these specific subgroups ranged from 0.69 to 0.72 , which could support the argument that perhaps in specific subgroups of patients the use of CDKi + ET might be cost effective.

Two main questions remain unanswered: is there any difference between each CDKi and what would be the optimal sequence of CDKi in the treatment of HR+/HER2- aBC.

Without a randomized controlled trial to compare the three FDA-approved CDKi head to head, it is uncertain if there is any significant difference between the efficacies of these agents - albeit, these agents seem to have similar efficacies. It seems as though the biggest differences between these drugs lies within their adverse event profiles due to varying molecular structures and off-target interactions [1].

In an indirect analysis of six trials including 3743 patients treated with either palbociclib, ribociclib or abemaciclib all concurrently with an aromatase inhibitor, PFS, ORR and grade 3-4 toxicities were reported. Generally, all CDKi provided similar benefits. The HR for PFS was calculated comparing palbociclib, to ribociclib and was found to be 1.04 (95\% CI: 0.73-1.46), 1.07 (95\% CI: 0.75-1.54) for palbociclib to abemaciclib and 1.04 (95\% CI: 0.71-1.52) 
for ribociclib to abemaciclib [18]. A similar calculation was performed for ORR and found to be 0.82 (95\% CI: 0.6-1.09) for palbociclib, to ribociclib, 0.87 (95\% CI: 0.63-1.19) for palbociclib to abemaciclib and 1.06 (95\% CI: 0.77-1.47) for ribociclib to abemaciclib [18]. There were similar HR and PFS profiles among the three agents. In terms of grade 3-4 toxicities, the most common toxicities were leukopenia, neutropenia, anemia, AST/ALT elevations and diarrhea. The only difference in toxicity profile was found in the palbociclib cohort compared with abemaciclib, which showed less grade 3-4 diarrhea in the palbociclib cohort [18].

In our pooled meta-estimate, the magnitude of OS gain seems to be the same in first- or second-line treatment (around 20\%), although extended follow-up of previously published studies and further studies (especially crossover trials) should clarify which would be the best sequence of CDKi in the treatment of HR+/HER2- aBC.

\section{Supplementary data}

To view the supplementary data that accompany this paper please visit the journal website at:

www.futuremedicine.com/doi/suppl/10.2217/fon-2019-0044

\section{Financial \& competing interests disclosure}

JJ Adashek has nothing to disclose. PN Aguiar Jr reports speaker fees from Merck Co., outside the submitted work. GL Lopes Jr reports advisory role from Pfizer, research funds from Merck Co., research funds from EMD Serono, research funds from Astra Zeneca, research funds from Blueprint Medicines, research funds from Tesaro, research funds from Bavarian Nordic, research funds from Novartis, research funds from G1 Therapeutics, outside the submitted work. RA de Mello: advisory board consultant for Zodiac, MSD, Pfizer. Speaker fee from Astra Zeneca, Novartis. Honoraria from National Medical Research Council, Singapore, and National Science Centre, Krakow, Poland. Research grant from Brazilian National Development Council for Science and Technology (CNPQ) number 402621/2016-6. The authors have no other relevant affiliations or financial involvement with any organization or entity with a financial interest in or financial conflict with the subject matter or materials discussed in the manuscript apart from those disclosed.

No writing assistance was utilized in the production of this manuscript.

\section{References}

1. Fernandes MT, Adashek JJ, Noia Barreto CM et al. A paradigm shift for the treatment of hormone receptor-positive, human epidermal growth factor receptor 2-negative (HR+/HER2-) advanced breast cancer: a review of CDK inhibitors. Drugs Context 7, 1-6 (2018).

2. DeVita VT Jr, Lawrence TS, Rosenberg SA. DeVita, Hellman, and Rosenberg's Cancer: Principles and Practice of Oncology (10th Edition). Lippincott Williams, PA, USA (2014).

3. Abraham J, Coleman R, Elias A et al. Use of cyclin-dependent kinase (CDK) 4/6 inhibitors for hormone receptor-positive, human epidermal growth factor receptor 2-negative, metastatic breast cancer: a roundtable discussion by The Breast Cancer Therapy Expert Group (BCTEG). Breast Cancer Res. Treat. 171(1), 11-20 (2018).

4. Shah AN, Cristofanilli M. The growing role of CDK4/6 inhibitors in treating hormone receptor-positive advanced breast cancer. Curr. Treat. Options Oncol. 18(1), 6 (2017).

5. Finn RS, Crown JP, Lang I et al. The cyclin-dependent kinase 4/6 inhibitor palbociclib in combination with letrozole versus letrozole alone as first-line treatment of oestrogen receptor-positive, HER2-negative, advanced breast cancer (PALOMA-1/TRIO-18): a randomised Phase II study. Lancet. Oncol. 16(1), 25-35 (2015).

6. Finn RS, Martin M, Rugo HS et al. Palbociclib and letrozole in advanced breast cancer. N. Engl. J. Med. 375(20), 1925-1936 (2016).

7. Cristofanilli M, Turner NC, Bondarenko I et al. Fulvestrant plus palbociclib versus fulvestrant plus placebo for treatment of hormone-receptor-positive, HER2-negative metastatic breast cancer that progressed on previous endocrine therapy (PALOMA-3): final analysis of the multicentre, double-blind, Phase 3 randomised controlled trial. Lancet Oncol. 17(4), 425-439 (2016).

8. Hortobagyi GN, Stemmer SM, Burris HA et al. Ribociclib as first-line therapy for HR-positive, advanced breast cancer. N. Engl. J. Med. 375(18), 1738-1748 (2016).

9. Slamon DJ, Neven P, Chia $S$ et al. Phase III randomized study of ribociclib and fulvestrant in hormone receptor-positive, human epidermal growth factor receptor 2-negative advanced breast cancer: MONALEESA-3. J. Clin. Oncol. 36(24), 2465-2472 (2018).

10. Tripathy D, Im S-A, Colleoni M et al. Ribociclib plus endocrine therapy for premenopausal women with hormone-receptor-positive, advanced breast cancer (MONALEESA-7): a randomised Phase 3 trial. Lancet. Oncol. 19(7), 904-915 (2018).

11. Sledge GW, Toi M, Neven P et al. MONARCH 2: abemaciclib in combination with fulvestrant in women with HR+/HER2-advanced breast cancer who had progressed while receiving endocrine therapy. J. Clin. Oncol. 35(25), 2875-2884 (2017).

12. Goetz MP, Toi M, Campone M et al. MONARCH 3: abemaciclib as initial therapy for advanced breast cancer. J. Clin. Oncol. 35(32), 3638-3646 (2017). 
13. Turner NC, Slamon DJ, Ro J et al. Overall survival with palbociclib and fulvestrant in advanced breast cancer. N. Engl. J. Med. 379(20), 1926-1936 (2018)

14. Finn RS, Crown J, Lang I, Boer K, Bondarenko I, Kulyk SO. Overall survival results from the randomized Phase II study of palbociclib $(\mathrm{P})$ in combination with letrozole $(\mathrm{L})$ vs letrozole alone for frontline treatment of ER+/HER2- advanced breast cancer (PALOMA-1; TRIO-18). Presented at: 2017 ASCO Annual Meeting, IL, USA, 1 June 2017.

15. Hortobagyi GN, Stemmer SM, Burris HA et al. Updated results from MONALEESA-2, a Phase III trial of first-line ribociclib plus letrozole versus placebo plus letrozole in hormone receptor-positive, HER2-negative advanced breast cancer. Ann. Oncol. 29(7), 1541-1547 (2018).

16. GoodRx. Palbociclib prices and palbociclib coupons. www.goodrx.com/palbociclib

17. Aguiar P Jr, Barreto CMN, Bychkovsky BL, Lopes GL Jr. Cost-effectiveness studies in oncology. In: Methods and Biostatistics in Oncology. Araújo RL, Riechelman RP (Eds). Springer, Cham, 257-266 (2018). https://link.springer.com/chapter/10.1007\%2F978-3-319-71324-3_16

18. Messina C, Cattrini C, Buzzatti G et al. CDK4/6 inhibitors in advanced hormone receptor-positive/HER2-negative breast cancer: a systematic review and meta-analysis of randomized trials. Breast Cancer Res. Treat. 172(1), 9-21 (2018). 